\title{
eJRIEPS
}

Ejournal de la recherche sur l'intervention en éducation physique et sport

Hors-série $N^{\circ} 3 \mid 2019$

Le numérique en EPS

\section{Impact des technologies numériques sur la motivation des élèves en éducation physique au sein du style d'enseignement par la découverte guidée}

Cédric Roure

\section{(2) OpenEdition}

Journals

Édition électronique

URL : http://journals.openedition.org/ejrieps/3570

DOI : 10.4000/ejrieps.3570

ISSN : 2105-0821

Éditeur

ELLIADD

Référence électronique

Cédric Roure, «Impact des technologies numériques sur la motivation des élèves en éducation

physique au sein du style d'enseignement par la découverte guidée », eJRIEPS [En ligne], Hors-série N 3 | 2019, mis en ligne le 01 décembre 2019, consulté le 18 février 2020. URL : http:// journals.openedition.org/ejrieps/3570; DOI : 10.4000/ejrieps.3570

La revue eJRIEPS est mise à disposition selon les termes de la Creative Commons Attribution 4.0 International License. 


\section{Impact des technologies numériques sur la motivation des élèves en éducation physique au sein du style d'enseignement par la découverte guidée}

Cédric Roure*

* Unité d'Enseignement et de Recherche en Éducation Physique et Sport, Haute École Pédagogique, Canton de Vaud, Lausanne, Suisse.

\section{Résumé}

De nombreuses recherches ont montré les effets bénéfiques des technologies numériques sur la motivation des élèves, notamment à partir du moment où elles étaient supportées par une approche pédagogique. L'objectif de cette étude était ainsi d'étudier l'impact d'un style d'enseignement centré sur l'élève couplé à des technologies numériques sur la motivation des élèves, tant sur le plan contextuel que situationnel. Les participants étaient 118 élèves $d u$ secondaire ( $M=16.39, E T=0.64,100 \%$ de filles, 16-18 ans), répartis de manière aléatoire dans deux groupes : (a) un groupe expérimental ayant suivi un cycle d'Acrogym avec le style de la découverte guidée et les technologies numériques, et (b) un groupe contrôle ayant suivi le même cycle d'Acrogym avec le style de la commande sans technologies numériques. Les élèves ont répondu à des échelles de mesure de la motivation contextuelle avant et après l'implémentation du cycle, et à des questionnaires relatifs à leur motivation situationnelle au début et à la fin du cycle. Les résultats montrent que les scores obtenus par le groupe expérimental concernant la motivation contextuelle et situationnelle sont plus élevés que ceux du groupe contrôle. De plus, la motivation situationnelle du groupe expérimental a davantage évolué que celle du groupe contrôle. En conclusion, l'utilisation des technologies numériques couplée au style d'enseignement par la découverte guidée semble être une option intéressante pour développer la motivation des élèves, et plus largement leur engagement en éducation physique.

Mots clés : technologies numériques, découverte guidée, Education physique, motivation. 


\section{eJRIEPS Hors Série 3 Décembre 2019}

Abstract

Many researchers have demonstrated the positive effects of digital technologies on students' motivation when they were supported by a pedagogical approach. The purpose of this study was to examine the impact of a student-centred teaching style, coupling with digital technologies, on students' contextual and situational motivation. The participants were 118 secondary school students $(M=16.39, S D=0.64,100 \%$ girls, $16-18$ years) who were randomly allocated to two groups: (a) an experimental group in which students practiced an Acrogym unit within the guided discovery teaching style and with digital technologies, and (b) a control group in which students practiced the same Acrogym unit within the command teaching style and without digital technologies. Students responded to questionnaires related to their contextual motivation pre and post-implementation, and to their situational motivation at the beginning and at the end of the Acrogym unit. The results showed that students in the experimental group reported higher scores for their contextual and situational motivation. In addition, the situational motivation of the experimental group evolved more than those of the control group. In conclusion, it seems that the digital technologies within the guided discovery teaching style are interesting to develop students' motivation and engagement in physical education.

Key words : digital technologies, guided discovery, physical education, motivation.

\section{Introduction}

La génération actuelle d'adolescents et de jeunes adultes est très impliquée dans les réseaux sociaux, les technologies numériques et les jeux vidéo (Ream, Elliott \& Dunlap, 2013). D'après Ream et al. (2013), cette interaction avec les technologies numériques et les réseaux sociaux commence dès l'enfance pour se continuer à l'âge adulte. Un rapport sur les comportements de santé des enfants et adolescents a d'ailleurs montré que $91.5 \%$ d'entre eux passaient plus de deux heures par jour devant un écran (Health Behaviour in School-Aged Children's report ; Godeau, Navarro \& Arnaud, 2012). Dans le même temps, au niveau de la sphère éducative, les technologies numériques n'ont cessé de grandir au point de devenir des outils courants dans les classes de plusieurs pays (Kretschmann, 2015 ; Sung, Chang \& Liu, 2016). Même si de nombreuses études ont participé à la promotion et à l'attractivité des technologies numériques dans le contexte éducatif, Selwyn (2014) a informé les chercheurs qu'un écart persistait toujours entre les discours sur la 


\section{eJRIEPS Hors Série 3 Décembre 2019}

manière dont les technologies numériques devraient être utilisées et la réalité de leur utilisation en éducation. Dans le même registre, Weir et Connors (2009) avaient déjà mis en évidence que les technologies numériques n'étaient pas suffisamment exploitées pour impacter les pratiques pédagogiques des enseignants et affecter positivement les apprentissages des élèves. Cette relation complexe entre la sphère éducative et les technologies numériques n'est pas surprenante dans la mesure où la littérature a déjà montré un rapport paradoxal des enseignants envers ces technologies. Alors que certains professeurs sont des utilisateurs enthousiastes des technologies numériques dans leurs classes, d'autres sont plus méfiants voire résistent notamment car ils ne se sentent pas suffisamment compétents ou ne ressentent pas de supports externes pour pleinement intégrer les technologies au sein de leurs leçons (Fullan, 2013 ; Palao, Hastie, Cruz \& Ortega, 2015). Dans ces circonstances, l'utilisation des technologies numériques dans les classes doit être questionnée à partir d'une approche pédagogique pour clairement identifier les bénéfices potentiels sur les apprentissages et la motivation des élèves (Casey, Goodyear \& Armour, 2017).

\section{Technologies numériques et pédagogie en éducation physique}

Comme de nombreuses études en éducation (Stillar, 2012), l'utilisation des technologies numériques dans les classes d'éducation physique (EP) a souvent révélé des résultats positifs sur les apprentissages des élèves en comparaison d'une pédagogie «traditionnelle ». Par exemple, une recherche en danse a démontré que l'utilisation avancée et innovante des technologies numériques produisait des effets positifs sur les capacités cognitives des élèves en comparaison d'un enseignement classique fondé sur le processus d'imitation et de reproduction (Dania, Hatziharistos, Koutsouba \& Tyrovola, 2011). De plus, dans une étude quasi-expérimentale en gymnastique, des élèves assignés à un enseignement ancré sur les technologies numériques (enregistrement vidéo, visionnage en ligne...) ont reporté une plus grande perception d'autonomie et une plus grande motivation autodéterminée en comparaison d'élèves assignés à un enseignement traditionnel (Legrain, Gillet, Gernigon \& Lafrenière, 2015). Dans le même registre, Goodyear, Casey et Kirk (2014) avaient émis l'hypothèse que les filles étaient désengagées en EP principalement à cause d'une approche pédagogique traditionnelle et transmissive. En promouvant l'utilisation des technologies numériques au sein d'une pédagogie centrée sur l'élève, leur recherche a permis l'implication des élèves dans différents rôles tels que 


\section{eJRIEPS Hors Série 3 Décembre 2019}

celui de coach ou encore de productrice de vidéo. Leurs résultats ont montré une augmentation de l'engagement des filles dans les cours d'EP, même si leur participation active dans certains rôles (productrice de vidéo) n'a pas été tout le temps transférée en activité motrice. En conclusion, les recherches ont clairement montré les bénéfices des technologies numériques en EP lorsque celles-ci étaient intégrées dans une approche pédagogique centrée sur l'élève.

En parallèle de ces études, d'autres recherches se sont intéressées aux effets de certaines technologies numériques sur les apprentissages et la motivation des élèves, sans forcément les intégrer dans des approches pédagogiques particulières. Dans ce domaine, l'une des technologies les plus utilisées dans les classes d'EP est le feedback vidéo (Banville \& Polifko, 2009; O'Loughlin, Chroinin \& O'Grady, 2013 ; Weir \& Connors, 2009). Cette technologie permet au professeur de donner des feedback objectifs et immédiats sur les exécutions des élèves de par l'usage de tablettes tactiles (Harris, 2009). Les études ont montré l'impact positif du feedback vidéo sur les apprentissages des élèves en volleyball et en football, à la fois sur les habiletés techniques (Raiola, Parisi \& Napolitano, 2014) et sur la performance collective (Harvey \& Gittins, 2014). De plus, Casey et Jones (2011) ont démontré que les élèves utilisant les feedback vidéo développaient une compréhension plus fine des habiletés de lancer et attraper et avaient un meilleur engagement dans les leçons en EP. Complémentairement aux études ayant investigué les effets des feedback vidéo sur la motivation et les apprentissages des élèves (O'Loughlin et al., 2013), d'autres travaux se sont intéressés à l'utilisation de différents types de feedback vidéo. Les résultats convergent pour montrer que le feedback vidéo a plus d'effets positifs lorsqu'il est accompagné d'un feedback du professeur ou des camarades (Huff \& Schwan, 2012 ; Sung et al., 2016). A titre d'illustration, Lu-Ho, Iwen et Gwo-Jen (2016) ont comparé les effets de trois conditions de feedback vidéo sur la motivation des élèves: (a) vidéos avec commentaires des camarades, (b) vidéos avec évaluations des camarades, et (c) vidéos avec commentaires et évaluations des camarades. Leurs résultats montrent que la dernière condition a eu le plus d'impact sur la motivation intrinsèque des élèves et leur sentiment d'efficacité personnel. L'analyse du contenu des feedbacks délivrés aux élèves montre une meilleure qualité de ceux-ci lorsqu'il y avait un couplage entre commentaires et évaluations. Pour conclure, les recherches montrent clairement que les effets positifs des technologies numériques en EP sont atteints lorsque les enseignants utilisent certaines formes de technologies, comme les feedback vidéo ou les vidéos en ligne et quand ceci est supporté par une approche pédagogique centrée sur l'élève, où les commentaires et évaluations de 


\section{eJRIEPS Hors Série 3 Décembre 2019}

ceux-ci sont sollicités. Pour autant, qu'entend-on par une approche pédagogique centrée sur l'élève ? Et quels sont les effets de ces approches sur la motivation des élèves ?

\section{Les styles d'enseignement et leurs effets sur la motivation des élèves en EP}

Afin de clairement identifier ce que pourrait être une approche pédagogique centrée sur l'élève, la référence au spectre des styles d'enseignement de Mosston et Ashworth (2002) s'avère être relativement intéressante. En effet, ce spectre est un continuum de styles d'enseignement catégorisés en fonction des décisions prises par le professeur ou les élèves durant les leçons d'EP. Ce continuum s'étend d'approches centrées sur le professeur à des approches centrées sur les élèves. A une première extrémité du continuum, nous retrouvons le style le plus centré sur l'enseignant qui est le style de la commande. Dans celui-ci, le professeur prend toutes les décisions au cours la leçon (définit les consignes, exécute les démonstrations, choisit l'organisation spatiale des élèves, corrige les élèves, détermine le nombre de répétitions...). A l'autre extrémité se trouve le style le plus centré sur l'élève qui est le style d'enseignement autonome. Dans ce dernier, l'élève prend toutes les décisions et responsabilités quant au processus d'apprentissage. Entre ces deux extrêmes, d'autres styles d'enseignement existent où les responsabilités du professeur et des élèves évoluent. D'une manière simplifiée, les auteurs classent l'ensemble des styles d'enseignement (au nombre de 11) en deux clusters: production et reproduction. Dans le cluster de la reproduction, où l'on retrouve les styles prioritairement centrés sur l'enseignant, l'objectif des élèves est de reproduire ou de se souvenir des habiletés motrices ou des connaissances enseignées. A l'inverse, dans le cluster de la production, où l'on retrouve les styles prioritairement centrés sur l'élève, l'objectif est de découvrir des nouvelles connaissances ou des solutions à un problème.

Plusieurs études se sont attachées à comparer les effets de plusieurs styles d'enseignement sur la motivation des élèves. Par exemple, Morgan, Kingston et Sproule (2005) ont comparé trois styles : (a) le style couplé de la commande et de la pratique, (b) le style d'enseignement avec les pairs (observations, tutorat), et (c) le style de la découverte guidée (expérimenter et trouver des solutions à un problème, dévolution). Leurs résultats ont montré que les élèves soumis aux styles d'enseignement avec les pairs et de la découverte guidée percevaient un climat d'apprentissage orienté vers la maîtrise alors que ceux soumis aux styles de la commande et de la pratique percevaient un climat d'apprentissage orienté vers la performance. De plus, les styles d'enseignements davantage centrés sur les élèves (style 


\section{eJRIEPS Hors Série 3 Décembre 2019}

par les pairs et découverte guidée), étaient plus soutenant de l'autonomie et de la compétence de l'élève. Enfin, lors d'entretiens semi-dirigés et de focus group, les élèves ont exprimé leur plaisir d'essayer différentes solutions pour résoudre un problème lors du style de la découverte guidée. Ces résultats ont été confirmés dans une recherche similaire de Salvara, Jess, Abbott et Bognar (2006) ayant comparé des styles de la production avec des styles de la reproduction. Les élèves ayant suivi des leçons dans des styles de la reproduction avaient perçu un climat de classe soutenant l'ego et étaient plus enclins à poursuivre des buts d'accomplissements orientés vers l'ego et la performance. Et ceux soumis aux styles de la production poursuivaient des buts d'accomplissements de maîtrise et percevaient un climat de classe soutenant la tâche. Par la suite, cette tendance a de nouveau été confirmée par Papaioannou, Theodosiou, Pashali et Digelidis (2012) et Pitsi, Digelidis et Papaioannou (2015) dans la mesure où des élèves ayant participé à des leçons d'EP avec le style d'enseignement autonome (le plus centré sur l'élève) ont rapporté une plus grande motivation intrinsèque, des buts d'accomplissement de la maîtrise en comparaison d'élèves ayant suivis le style de la pratique ou de la commande. Enfin, deux autres études ont également confirmé les effets positifs des styles d'enseignement centrés sur les élèves pour développer leur motivation autodéterminée (Chatzipanteli, Digelidis \& Papaioannou, 2015 ; Kirby, Byra, Readdy \& Wallhead, 2015).

\section{Objectif de l'étude}

Les études recensées dans les paragraphes précédents montrent clairement les effets bénéfiques des technologies numériques sur la motivation des élèves. Ces effets sont également plus prononcés à partir du moment où les technologies numériques sont supportées par une approche pédagogique. De plus, d'autres études ont révélé que les styles d'enseignements centrés sur l'élève étaient de nature à favoriser le développement de leur motivation intrinsèque. Dès lors, il serait intéressant d'investiguer et de comprendre les effets des technologies numériques couplées à un style d'enseignement centré sur l'élève sur la motivation des élèves. Malheureusement, il n'existe aucune étude à ce jour, à notre connaissance, ayant investigué les effets d'une pédagogie centrée sur l'élève et couplée à des outils numériques sur les différentes formes de motivations des élèves (contextuelle et situationnelle). L'objectif de notre étude est ainsi d'étudier l'impact d'un style d'enseignement centré sur l'élève couplé à des technologies numériques sur la motivation des élèves, tant sur le plan contextuel que situationnel. 


\section{eJRIEPS Hors Série 3 Décembre 2019}

Pour mener à bien cette recherche, nous avons retenu le style de la découverte guidée au sein d'un cycle de sept leçons d'Acrogym, auquel nous avons associé certaines technologies numériques. Tout d'abord, nous avons choisi ce style d'enseignement car il est l'un des premiers styles appartenant au cluster de la production, où les élèves ont une grande part de responsabilité dans les apprentissages (Mosston \& Ashworth, 2002). Ce style de la découverte guidée se caractérise par la présentation aux élèves d'un problème à résoudre, suivi d'un jeu de questions / réponses entre le professeur et les élèves pour arriver à déterminer certaines solutions au problème initial. L'objectif du professeur est de questionner les élèves pour progressivement les amener vers le comportement attendu, c'est-à-dire celui qui leur permettra de trouver une solution. A l'intérieur de ce style d'enseignement, les technologies numériques suivantes ont été utilisées : enregistrement de vidéo, feedback vidéo et commentaires (professeur et camarades) et postage de vidéo en ligne. Ce travail autour de la vidéo était employé pour soutenir le processus de résolution de problème. Au cours des sept leçons d'Acrogym, les élèves étaient confrontés aux problèmes inhérents à la création et construction de pyramides statiques et dynamiques. L'organisation de l'ensemble du cycle sera détaillée dans la partie méthode.

Enfin, l'évaluation de l'impact de ce style d'enseignement couplé aux technologies numériques sur la motivation des élèves a été opérée au travers de trois concepts théoriques. Tout d'abord, la motivation contextuelle a été approchée par l'intermédiaire de la théorie des buts d'accomplissements (Elliott \& McGregor, 2001) et la théorie de l'expectancy-value (Eccles \& Wigfield, 2002). II s'agit de deux théories largement reconnues au plan de la recherche et très utilisées en EP qui interrogent la motivation des élèves à un niveau relativement général correspondant à des traits stables de la personnalité (Korn \& Elliot, 2016 ; Xiang, Liu, McBride \& Bruene, 2011 ; Yli-Piipari \& Kokkonen, 2014). Quant à la motivation situationnelle, nous avons retenu la théorie de l'intérêt en situation, cette dernière ayant été spécifiquement élaborée pour interroger ce niveau d'analyse (Roure, Kermarrec \& Pasco, 2016 ; Roure \& Pasco, 2018a). II s'agit d'une théorie qui interroge la motivation des élèves à un niveau spécifique correspondant à des états psychologiques qui peuvent évoluer à court et moyen terme.

Deux hypothèses de recherche ont été formulées en lien avec les précédents résultats obtenus dans la littérature. Premièrement, nous nous attendons à ce que le groupe expérimental obtienne des scores plus élevés au niveau de la motivation contextuelle à la fin de l'implémentation, ainsi qu'au niveau de la motivation situationnelle au début et à la fin 


\section{eJRIEPS Hors Série 3 Décembre 2019}

du cycle d'Acrogym. Et deuxièmement, nous pensons que la motivation situationnelle changera davantage chez le groupe expérimental par rapport au groupe contrôle.

\section{Méthodologie}

\subsection{Participants}

Cent-dix-huit élèves d'un établissement scolaire, situé en périphérie d'une agglomération moyenne de 70000 habitants en Belgique, ont participé à cette étude $(M=16.39, E T=0.64$, 100\% de filles, 16-18 ans). II s'agissait d'un établissement ayant un score plutôt élevé (14/20) pour l'indice socioéconomique des élèves (Fédération Wallonie-Bruxelles, 2015). De plus, cet établissement était détenteur du label «école numérique » l'inscrivant dans une dynamique de développement des technologies dans un contexte pédagogique. Les élèves appartenaient à cinq classes des deux derniers niveaux de l'Ecole secondaire en Wallonie. Ils étaient en moyenne entre 22 et 26 par classe. Les élèves étaient toutes de sexe féminin dans la mesure où l'EP est non mixte en Fédération Wallonie-Bruxelles et que l'enseignante impliquée dans l'étude n'avait que des classes avec des filles. Les élèves ont été répartis de manière aléatoire dans deux groupes : (a) un groupe expérimental constitué de trois classes et 81 élèves ayant suivi le cycle d'Acrogym avec le style de la découverte guidée et les technologies numériques, et (b) un groupe contrôle constitué de deux classes et 37 élèves ayant suivi le cycle d'Acrogym avec le style de la commande sans technologies numériques. L'enseignante impliquée dans l'étude était certifiée à plein-temps dans l'établissement et avait 15 ans d'expérience dans l'enseignement de l'EP. La direction de l'établissement et l'enseignante d'EP ont donné leur accord à la conduite de cette étude. Les parents des élèves concernés ont été informés des objectifs de l'étude et un formulaire de consentement leur a été demandé. Tous ont autorisé leurs enfants à participer à l'étude.

\subsection{Conception du cycle d'Acrogym}

L'enseignante d'EP impliquée dans cette étude et responsable des cinq classes d'élèves, en collaboration avec le chercheur, a créé un cycle de sept leçons d'Acrogym. La compétence finale visée était la création d'un enchaînement dynamique de minimum cinq pyramides (duo, trio et quatuor) avec des liaisons chorégraphiques. Les contenus d'enseignement des leçons portaient sur les principes de création et de construction des pyramides (statiques et dynamiques) ainsi que sur les éléments de liaison chorégraphique. 


\section{eJRIEPS Hors Série 3 Décembre 2019}

En lien avec l'objectif de l'étude, les deux groupes constitués (expérimental et contrôle) avaient strictement les mêmes contenus d'enseignement. La seule différence entre les deux groupes portait sur le style d'enseignement retenu (style de la découverte guidée pour le groupe expérimental vs style de la commande pour le groupe contrôle) et sur l'usage ou non des technologies numériques. Dans le groupe expérimental, les élèves étaient placés par groupe de quatre à cinq avec une tablette tactile par groupe, connectée à internet. Lors de chaque leçon, les élèves étaient invités à utiliser la tablette tactile au sein du style de la découverte guidée. Etant donné que le principe organisateur de ce style d'enseignement repose sur la présentation d'un problème à résoudre, suivi d'une phase de recherche et expérimentation de solutions, les technologies numériques sont venues soutenir cette pédagogie. Ainsi, la tablette était utilisée pour effectuer des recherches sur les différentes manières de créer et construire des pyramides, pour se filmer et avoir un feedback vidéo lorsque les élèves tentaient une pyramide, pour poster des vidéos en ligne afin de recevoir des feedbacks et commentaires des camarades, et enfin pour regarder des vidéos d'autres élèves dans l'optique d'émettre des feedbacks et commentaires. A titre d'illustration, lors de la deuxième leçon du cycle, les élèves étaient invités, après avoir cherché des principes de construction de pyramides, à créer des tutoriels sur la pyramide de leur choix et les poster en ligne. Ces tutoriels devaient permettre à un autre groupe d'élèves de comprendre les principes de la pyramide et de pouvoir la refaire à leur tour. Dans cet exemple, le problème à résoudre par les élèves était de trouver les principes sous-jacents à la construction d'une pyramide et être capable de les expliquer et les mettre en forme dans un tutoriel vidéo. Finalement, dans un objectif de soutien au style de la découverte guidée, la professeure questionnait régulièrement les élèves pour les amener progressivement à trouver des solutions au problème initial. Par exemple, toujours dans cette deuxième leçon, la professeure interrogeait les élèves pour savoir comment d'autres élèves pourraient comprendre les consignes prévues pour le tutoriel vidéo (A votre avis, comment vos camarades vont comprendre vos consignes ? Est-ce que si vous receviez les mêmes consignes, vous pourriez créer la pyramide ?).

\subsection{Fidélité de l'intervention}

Afin de s'assurer de la fidélité de l'intervention pour le groupe expérimental, un guide a été conçu par le chercheur au niveau du style de la découverte guidée et des outils numériques. Ce guide a été expliqué et discuté avec la professeure. II était en lien avec le cycle de sept 


\section{eJRIEPS Hors Série 3 Décembre 2019}

leçons élaborées en Acrogym et comprenait quatre éléments : présentation des objectifs de leçons sous la forme d'un problème à résoudre, utilisation de la tablette tactile pour aider à la résolution du problème, utilisation de feedback interrogatifs pour soutenir l'expérimentation et la recherche des solutions de la part des élèves, validation des solutions au problème en fin de séance. Avant de conduire cette étude, l'enseignante s'était entraînée au style de la découverte guidée avec une autre classe (qui ne participait pas à la recherche) durant quatre séances d'Acrogym. Après chaque séance, le chercheur donnait des feedback et conseils à l'enseignante en appui avec le guide créé. De plus, la fréquence d'utilisation des tablettes tactiles était vérifiée afin de s'assurer que les élèves avaient un taux d'utilisation optimal. A l'issue de ces quatre séances, l'enseignante avait atteint un niveau suffisant pour démarrer l'implémentation.

Pendant l'expérimentation, une grille d'analyse a été élaborée sur la base des quatre éléments constituant le guide pour le style de la découverte guidée. Pour chaque élément, un codage a été effectué pour vérifier la fidélité de l'implémentation $(A=$ élément correctement maitrisé, $B=$ élément partiellement maitrisé, $C=$ élément absent). Cette grille d'analyse a été utilisée sur quatre leçons du cycle (leçon 1, 2, 4 et 6) qui ont été filmées en plus d'un enregistrement audio du discours de l'enseignante.

\subsection{Mesures de la motivation des élèves}

Théorie des buts d'accomplissements. La version française en 12 items du "French Achievement Goals Questionnaire for Sport and Exercise (FAGQSE) » (Riou et al., 2012) a été utilisée pour mesurer les buts d'accomplissement des élèves. Les quatre variables mesurées sont celles du modèle $2 \times 2$ des buts d'accomplissement (Elliot \& McGregor, 2001), à savoir : maîtrise-approche (e.g. " Je cherche à réaliser le mieux possible ce que je dois faire "), maîtrise-évitement (e.g. " Je cherche à éviter de faire mal les choses »), performance-approche (e.g. "Mon but est d'être meilleur que les autres ») et performanceévitement (e.g. " Je cherche à éviter d'être moins bon que les autres »). Chacune des quatre variables a été mesurée au moyen de trois items, répartis de manière aléatoire, sur une échelle de Likert allant de 1 (= «Pas du tout d'accord ") à 5 (= «Tout à fait d'accord "). La validité du questionnaire pour des élèves en EP a été établie au moyen d'analyses factorielles confirmatoires et exploratoires (NNFI $=.99, \mathrm{CFI}=.99$ et RMSEA $=.053$ ). La consistance interne (alpha de Cronbach) de chaque but a également été mesurée pour les élèves en EP avec des valeurs allant de .73 à .91. 


\section{eJRIEPS Hors Série 3 Décembre 2019}

Théorie de l'expectancy-value. La version française en 17 items du « French ExpectancyValue Questionnaire in Physical Education (FEVQ-PE) 》 (Roure \& Lentillon-Kaestner, 2018) a été utilisée pour mesurer les espérances de succès et valeurs des tâches des élèves. Ce questionnaire se compose de six items pour mesurer les espérances de succès (e.g. " Je pense que je suis bon en EP »), de quatre items pour mesurer l'importance (e.g. «C'est important pour moi de réussir en EP »), de quatre items pour mesurer l'intérêt (e.g. "Le temps passe vite lorsque je suis en cours d'EP»), et de trois items pour mesurer l'utilité (e.g. «Ce que j'ai appris en EP me servira pour d'autres matières ou en dehors de l'Ecole »). Les différentes variables ont été mesurées sur une échelle de Likert allant de 1 (= «Pas du tout d'accord 》) à 5 (= "Tout à fait d'accord »). La validité du questionnaire pour des élèves en EP a été établie au moyen d'analyses factorielles confirmatoires et exploratoires $(\mathrm{NNFI}=.94, \mathrm{CFI}=.96$ et RMSEA $=.06)$. La consistance interne (alpha de Cronbach) de chaque but a également été mesurée pour les élèves en EP avec des valeurs allant de .80 à .93.

Théorie de l'intérêt en situation. L'intérêt en situation (IS) des élèves a été mesuré en utilisant l'échelle de mesure de l'IS en EP (Roure, Pasco \& Kermarrec, 2016). Celle-ci se compose de 15 énoncés sur lesquels les élèves se prononcent à l'aide d'une échelle de Likert en 5 points allant de 1 (= «Pas du tout d'accord ») à 5 (= «Tout à fait d'accord »). L'échelle comprend trois énoncés pour chacune des cinq dimensions de l'IS : le plaisir instantané ( Ce que nous avons fait était agréable pour moi »), l'intention d'exploration (« J'ai cherché à analyser et à mieux saisir ce que nous avons appris aujourd'hui »), la demande d'attention ("Ce que nous avons appris m'a demandé beaucoup d'attention »), le défi («Ce que nous avons appris était difficile à faire pour moi ») et la nouveauté (« $\mathrm{Ce}$ que nous avons fait aujourd'hui était nouveau pour moi »). Roure et al. (2016) ont établi la validité du construit de l'échelle de mesure de l'IS en langue Française en utilisant des analyses factorielles exploratoires et confirmatoires (GFI $=0.93, \mathrm{NFI}=0.93, \mathrm{RMSEA}=$ 0.06). Ils ont aussi rapporté la consistance interne (alpha de Cronbach) pour la nouveauté $(0,83)$, le défi $(0,77)$, la demande d'attention $(0,76)$, l'intention d'exploration $(0,79)$, le plaisir instantané $(0,84)$ et, l'intérêt total $(0,85)$.

\subsection{Recueil des données}

Les mesures concernant la motivation contextuelle des élèves, évaluée à partir de la théorie des buts d'accomplissements et de la théorie de l'expectancy-value, ont été recueillies avant 


\section{eJRIEPS Hors Série 3 Décembre 2019}

et après le cycle de sept leçons d'Acrogym. L'objectif de ces deux temps de mesures était de pouvoir quantifier les différences avant et après l'expérimentation sur deux construits motivationnels considérés comme relativement stables dans le temps. Quant à la motivation situationnelle, appréhendée au travers de l'IS, le questionnaire a été passé à la fin des leçons 1, 2, 5 et 6. L'objectif était ici d'identifier l'évolution de la motivation situationnelle des élèves en fonction de l'avancement des leçons d'Acrogym. Toutes les données ont été collectées par le chercheur sous la supervision de l'enseignante d'EP en charge des classes. Le chercheur a administré les questionnaires aux élèves et les a collectés directement après. Pour minimiser la tendance des élèves à donner des réponses socialement désirables, ils ont été encouragés à répondre honnêtement et ils ont été assurés que leurs réponses resteraient anonymes et confidentielles.

\subsection{Analyse des données}

Les scores des élèves aux items relatifs aux cinq dimensions de l'IS (la nouveauté, le défi, la demande d'attention, l'intention d'exploration et le plaisir instantané) ont été additionnés. De plus, nous avons retenu pour chaque dimension de l'IS la moyenne des scores obtenus aux leçons 1 et 2 , puis aux leçons 5 et 6 . Par conséquent, nous avions deux scores pour chaque dimension de l'IS reflétant la motivation situationnelle des élèves au début du cycle (leçons 1 et 2) et à la fin du cycle (leçons 5 et 6). Pour la théorie des buts d'accomplissement, la moyenne des scores des items relatifs aux quatre orientations motivationnelles a été retenue (maîtrise-approche, maîtrise-évitement, performance-approche et performanceévitement). De la même manière, les moyennes de scores ont été calculées pour la théorie de l'expectancy-value au niveau des quatre variables (espérances de succès, importance, intérêt et utilité). Pour tester nos hypothèses de recherche, les données ont été analysées en deux étapes : l'une centrée sur la motivation contextuelle des élèves et l'autre centrée sur leur motivation situationnelle. Premièrement, nous avons réalisé des MANOVA pré et post-implémentation afin de comparer les scores des élèves entre le groupe expérimental et le groupe contrôle au niveau de la théorie des buts d'accomplissements et de l'expectancy-value. Puis, nous avons continué les analyses à l'intérieur de chaque groupe par des MANOVA à mesures répétées pour analyser l'évolution de la motivation contextuelle des élèves entre le début et la fin de l'expérimentation. Deuxièmement, nous avons réalisé des MANOVA pour comparer les scores des élèves entre le groupe expérimental et le groupe contrôle au niveau des cinq dimensions de l'IS au début du cycle 


\section{eJRIEPS Hors Série 3 Décembre 2019}

(moyennes des leçons 1 et 2) et à la fin du cycle (moyennes des leçons 5 et 6 ). Par la suite, nous avons continué les analyses à l'intérieur de chaque groupe par des MANOVA à mesures répétées pour analyser l'évolution de la motivation situationnelle des élèves entre le début et la fin du cycle d'Acrogym. La version 23.0 de SPSS (SPSS Inc, Chicago, IL) a été utilisée pour réaliser ces analyses statistiques et nous avons retenu un seuil de significativité fixé à .05 .

\section{Résultats}

\subsection{Analyses préliminaires}

L'analyse des valeurs d'aplatissement (-.78 à -.14) et d'asymétrie (-.34 à .71) a révélé que toutes les données étaient normalement distribuées et aucun problème de multicolinéarité entre les variables n'a été trouvé. Les consistances internes des trois échelles de mesure étaient correctes avec des valeurs respectives pour les alphas de Chronbach comprises entre .79 et .92, à l'exception de la variable maîtrise-évitement où le coefficient n'était que de .66. Par conséquent, seule la variable maîtrise-évitement a été supprimée des analyses ultérieures. De plus, étant donné la nature hiérarchique des données (i.e. les élèves appartenaient à cinq classes différentes), la part de variance expliquée par le niveau de classe a été analysée pour les variables de l'IS. Cette part de variance a été calculée au moyen d'un coefficient de corrélation intra-classe (ICC) qui permet de déterminer si la variance des scores de l'IS des élèves est davantage due à l'appartenance aux classes qu'aux différences entre les élèves. Les résultats montrent que les ICC pour les cinq dimensions de l'IS étaient tous compris entre .011 et .038. Autrement dit, la part de variance dans les scores de l'IS des élèves expliquée par l'appartenance aux classes varie entre $1.1 \%$ et $3.8 \%$. Dans ces circonstances, selon Preacher, Zhang et Zyphur (2011), une analyse multi-niveaux (i.e. les élèves au niveau 1 et les classes au niveau 2) aurait été moins efficace qu'une analyse simple niveau car les ICC étaient tous inférieurs à .10 pour l'ensemble des variables étudiées. En conséquence, nous avons procédé à une analyse simple niveau (i.e. celui des élèves) pour la suite des analyses statistiques. Enfin, les résultats concernant la fidélité de l'implémentation, obtenus à partir de l'analyse de quatre leçons du cycle, ont révélé que l'enseignante avait obtenu des « $A$ » pour tous les éléments constitutifs de la grille d'analyse, indiquant un très bon niveau de fidélité au style de la découverte guidée ainsi qu'une utilisation optimale des tablettes tactiles par les élèves. 


\section{eJRIEPS Hors Série 3 Décembre 2019}

\subsection{Résultats pour la motivation contextuelle des élèves}

Les résultats de la MANOVA, pré-implémentation ne révèlent pas de différence significative entre les variables des buts d'accomplissements et de l'expectancy-value en fonction des deux groupes (Trace de Pillaï $=.07, F(7,110)=1.19, p=.32, \eta^{2}=.07$ ). Ces résultats montrent que le groupe expérimental et le groupe contrôle n'étaient pas différents au niveau de la motivation contextuelle avant de démarrer le cycle de sept leçons d'Acrogym. Par contre, à la fin de l'implémentation, les résultats de la MANOVA révèlent une différence significative entre les deux groupes (Trace de Pillaï $=.17, F(7,110)=3.20, p<.01, \eta^{2}=.17$ ). Plus précisément, des différences significatives apparaissent au niveau des variables de l'intérêt et de l'utilité, avec des scores plus élevés pour le groupe expérimental (voir le tableau 1).

Tableau I : Résultats descriptifs pour la motivation contextuelle à la fin de l'implémentation selon les deux groupes

\begin{tabular}{lccccccc}
\hline & \multicolumn{7}{c}{$\begin{array}{c}\text { Groupe } \\
\text { expérimental } \\
(n=81)\end{array}$} \\
& Variation & $M$ & $E T$ & \multicolumn{2}{c}{$\begin{array}{c}\text { Groupe } \\
\text { contrôle } \\
(n=37)\end{array}$} \\
\hline Maitrise-approche & $1-5$ & 3.70 & 0.84 & 3.68 & 0.81 & 0.13 & .00 \\
Performance-approche & $1-5$ & 2.21 & 1.26 & 2.32 & 1.19 & 0.20 & .00 \\
Performance-évitement & $1-5$ & 3.05 & 1.22 & 2.96 & 0.95 & 0.13 & .00 \\
Espérances de succès & $1-5$ & 3.25 & 0.74 & 3.36 & 0.65 & 0.57 & .01 \\
Importance & $1-5$ & 3.35 & 0.88 & 3.02 & 0.90 & 3.46 & .03 \\
Intérêt & $1-5$ & 3.33 & 0.75 & 2.88 & 0.76 & $9.05^{*}$ & .07 \\
Utilité & $1-5$ & 2.63 & 0.91 & 2.10 & 0.74 & $9.59^{*}$ & .08
\end{tabular}

Note. F: valeur du test; ${ }^{*} p<.01 ; \eta^{2}$ : taille d'effet.

Dans le but de comprendre quelle a été l'évolution de la motivation contextuelle dans les deux groupes entre le début et la fin de l'implémentation, des analyses de MANOVA à mesures répétées ont été conduites. Pour le groupe expérimental, les résultats ne révèlent aucune différence significative pour les variables des buts d'accomplissements et de l'expectancy-value entre le début et la fin de l'implémentation (Trace de Pillaï $=.15, F(7,74)$ $\left.=1.92, p=.08, \eta^{2}=.15\right)$. En d'autres termes, la motivation contextuelle du groupe expérimental est restée stable durant l'implémentation. Par contre, les résultats de la 


\section{eJRIEPS Hors Série 3 Décembre 2019}

MANOVA à mesures répétées pour le groupe contrôle démontrent une différence significative sur les mêmes variables entre le début et la fin de l'implémentation (Trace de Pillaï $\left.=.57, F(7,30)=5.76, p<.01, \eta^{2}=.57\right)$. Comme nous pouvons le voir dans le tableau 2 ci-dessous, les scores relatifs à la théorie de l'expectancy-value ont diminué pour ce groupe durant l'implémentation.

Tableau II : Résultats descriptifs pour l'évolution de la motivation contextuelle pour le groupe contrôle

\begin{tabular}{|c|c|c|c|c|c|c|c|}
\hline & & implém & tation & implém & tation & & \\
\hline & Variation & $M$ & $E T$ & $M$ & $E T$ & $F(7,30)$ & $\eta^{2}$ \\
\hline Maitrise-approche & $1-5$ & 3.89 & 0.77 & 3.68 & 0.81 & 3.09 & .08 \\
\hline Performance-approche & $1-5$ & 2.30 & 1.22 & 2.32 & 1.19 & 0.01 & .00 \\
\hline Performance-évitement & $1-5$ & 3.31 & 1.01 & 2.96 & 0.95 & 3.90 & .10 \\
\hline Espérances de succès & $1-5$ & 3.52 & 0.70 & 3.36 & 0.65 & $5.12^{*}$ & .13 \\
\hline Importance & $1-5$ & 3.34 & 0.92 & 3.02 & 0.90 & $6.34^{*}$ & .15 \\
\hline Intérêt & $1-5$ & 3.44 & 0.74 & 2.88 & 0.76 & $25.50^{\star}$ & .42 \\
\hline Utilité & $1-5$ & 2.72 & 0.60 & 2.10 & 0.74 & $18.07^{*}$ & .33 \\
\hline
\end{tabular}

Note. F: valeur du test; * $p<.01 ; \eta^{2}$ : taille d'effet.

Ces premiers résultats nous montrent que la différence relevée à la fin de l'implémentation entre les deux groupes au niveau de la motivation contextuelle peut s'expliquer par une diminution des scores au niveau de la théorie de l'expectancy-value chez le groupe contrôle, associée à un maintien de la motivation chez le groupe expérimental.

\subsection{Résultats pour la motivation situationnelle des élèves}

Les résultats de la MANOVA, au début du cycle (leçons 1 et 2), révèlent une différence significative entre les variables de l'IS en fonction des deux groupes (Trace de Pillaï $=.87$, $\left.F(5,112)=152.99, p<.01, \eta^{2}=.87\right)$. A l'exception du plaisir instantané, tous les scores des autres dimensions de l'IS sont plus élevés pour le groupe expérimental (voir le tableau 3). Les trois dimensions où la taille d'effet est importante sont la nouveauté, la demande d'attention et le défi. 


\section{eJRIEPS Hors Série 3 Décembre 2019}

Tableau III : Résultats descriptifs pour la motivation situationnelle au début du cycle selon les deux groupes

\begin{tabular}{|c|c|c|c|c|c|c|c|}
\hline & \multirow[b]{2}{*}{ Variation } & \multicolumn{2}{|c|}{$\begin{array}{c}\text { Groupe } \\
\text { expérimental } \\
(n=81)\end{array}$} & \multicolumn{2}{|c|}{$\begin{array}{l}\text { Groupe } \\
\text { contrôle } \\
(n=37)\end{array}$} & \multirow[b]{2}{*}{$F(5,112)$} & \multirow[b]{2}{*}{$\eta^{2}$} \\
\hline & & $M$ & $E T$ & $M$ & $E T$ & & \\
\hline Plaisir instantané & $3-15$ & 10.12 & 1.16 & 10.23 & 1.01 & 0.24 & .00 \\
\hline Intention d'exploration & $3-15$ & 8.21 & 1.38 & 7.43 & 0.99 & $9.32^{*}$ & .07 \\
\hline Demande d'attention & $3-15$ & 10.82 & 0.72 & 9.57 & 1.05 & $56.97^{\star}$ & .33 \\
\hline Nouveauté & $3-15$ & 10.23 & 0.73 & 6.21 & 1.07 & $571.26^{*}$ & .83 \\
\hline Défi & $3-15$ & 9.37 & 1.41 & 7.56 & 0.98 & $49.69^{*}$ & .30 \\
\hline
\end{tabular}

Note. F: valeur du test; * $p<.01 ; \eta^{2}$ : taille d'effet.

Les résultats de la MANOVA, à la fin du cycle (leçons 5 et 6), révèlent une différence significative entre les variables de l'IS en fonction des deux groupes (Trace de Pillaï $=.64$, $\left.F(5,112)=40.66, p<.01, \eta^{2}=.64\right)$. A la différence du début du cycle, les différences ne sont cette fois que significatives pour le plaisir instantané, l'intention d'exploration et la demande d'attention avec des scores plus élevés pour le groupe expérimental (voir le tableau 4). Les tailles d'effet sont importantes pour le plaisir instantané et l'intention d'exploration.

Tableau IV : Résultats descriptifs pour la motivation situationnelle à la fin du cycle selon les deux groupes

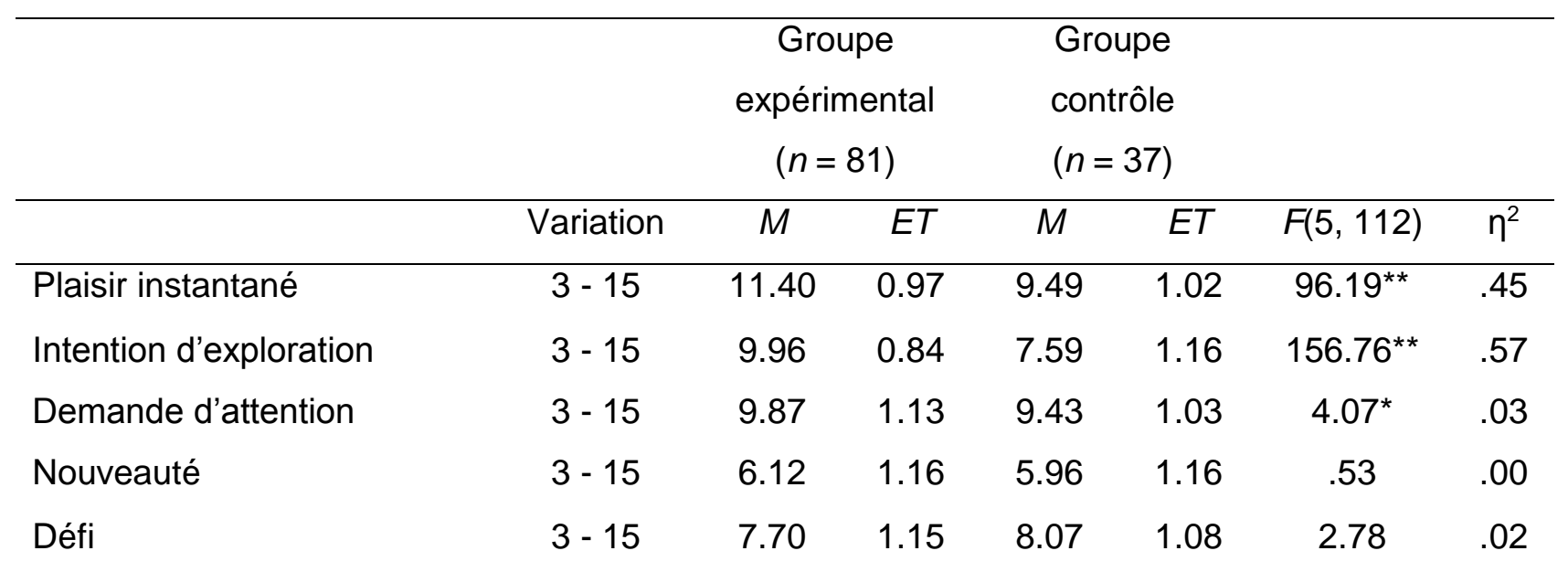

Note. F: valeur du test; ${ }^{*} p<.05 ;{ }^{* *} p<.01 ; \eta^{2}$ : taille d'effet. 


\section{eJRIEPS Hors Série 3 Décembre 2019}

Dans le but de comprendre quelle a été l'évolution de la motivation situationnelle dans les deux groupes entre le début et la fin du cycle, des analyses de MANOVA à mesures répétées ont été conduites. Pour le groupe contrôle, les résultats révèlent une différence significative pour les variables de l'IS entre le début et la fin du cycle (Trace de Pillaï $=.90$, $\left.F(5,32)=55.54, p<.01, \eta^{2}=.90\right)$. A l'exception de l'intention d'exploration, les autres dimensions de l'IS ont évolué au cours du cycle. Le plaisir instantané, la demande d'attention et la nouveauté ont diminué alors que le défi a augmenté (voir le tableau 5).

Tableau V : Résultats descriptifs pour l'évolution de la motivation situationnelle pour le groupe contrôle

Début du cycle Fin du cycle

\begin{tabular}{lccccccc}
\hline & Variation & $M$ & $E T$ & $M$ & $E T$ & $F(5,32)$ & $\eta^{2}$ \\
\hline Plaisir instantané & $3-15$ & 10.23 & 1.01 & 9.49 & 1.02 & $71.36^{* *}$ & .66 \\
Intention d'exploration & $3-15$ & 7.43 & 0.99 & 7.59 & 1.16 & 3.37 & .09 \\
Demande d'attention & $3-15$ & 9.57 & 1.05 & 9.43 & 1.03 & $5.61^{*}$ & .13 \\
Nouveauté & $3-15$ & 6.21 & 1.07 & 5.96 & 1.16 & $6.69^{*}$ & .16 \\
Défi & $3-15$ & 7.56 & 0.98 & 8.07 & 1.08 & $11.95^{\star *}$ & .25 \\
\hline
\end{tabular}

Note. F: valeur du test; ${ }^{*} p<.05 ;{ }^{* *} p<.01 ; \eta^{2}$ : taille d'effet.

Pour le groupe expérimental, les résultats révèlent une différence significative pour les variables de l'IS entre le début et la fin du cycle (Trace de Pillaï $=.94, F(5,76)=255.86, p<$ $\left..01, \eta^{2}=.94\right)$. Toutes les dimensions de l'IS ont évolué au cours du cycle. Le plaisir instantané et l'intention d'exploration ont augmenté tandis que la demande d'attention, la nouveauté et le défi ont diminué (voir le tableau 6). 
Tableau VI: Résultats descriptifs pour l'évolution de la motivation situationnelle pour le groupe expérimental

Début du cycle Fin du cycle

\begin{tabular}{lccccccc}
\hline & Variation & $M$ & $E T$ & $M$ & $E T$ & $F(5,76)$ & $\eta^{2}$ \\
\hline Plaisir instantané & $3-15$ & 10.12 & 1.16 & 11.40 & 0.97 & $53.09^{*}$ & .40 \\
Intention d'exploration & $3-15$ & 8.21 & 1.38 & 9.96 & 0.84 & $87.23^{*}$ & .52 \\
Demande d'attention & $3-15$ & 10.82 & 0.72 & 9.87 & 1.13 & $40.98^{*}$ & .34 \\
Nouveauté & $3-15$ & 10.23 & 0.73 & 6.12 & 1.16 & $974.20^{*}$ & .92 \\
Défi & $3-15$ & 9.37 & 1.41 & 7.70 & 1.15 & $59.47^{*}$ & .43
\end{tabular}

Note. F: valeur du test; ${ }^{\star} p<.01 ; \eta^{2}$ : taille d'effet.

Ces résultats montrent que le groupe expérimental a perçu davantage de plaisir instantané et d'intention d'exploration au fur et à mesure du cycle dans le même temps que la nouveauté et le défi diminuait. Conjugué à la baisse du plaisir instantané du groupe contrôle au cours du cycle, il est compréhensible que les différences entre les deux groupes sur le plan de la motivation situationnelle à la fin du cycle soient principalement basées sur le plaisir instantané et l'intention d'exploration. Initialement basées sur la nouveauté, le défi et la demande d'attention au début du cycle, les différences entre les deux groupes sur l'IS se sont donc progressivement dirigées vers le plaisir instantané et l'intention d'exploration.

\section{Discussion}

L'objectif de cette recherche était d'étudier l'impact d'un style d'enseignement centré sur l'élève couplé à des technologies numériques sur la motivation des élèves, tant sur le plan contextuel que situationnel. Au regard de la littérature disponible, deux hypothèses fondaient notre recherche : (1) le groupe expérimental devrait obtenir des scores plus élevés au niveau de la motivation contextuelle à la fin de l'implémentation, ainsi qu'au niveau de la motivation situationnelle au début et à la fin du cycle d'Acrogym, et (2) la motivation situationnelle devrait changer davantage chez le groupe expérimental par rapport au groupe contrôle. 


\section{eJRIEPS Hors Série 3 Décembre 2019}

\subsection{Un impact sur la motivation contextuelle et situationnelle des élèves}

Notre première hypothèse est validée puisque les scores obtenus par le groupe expérimental concernant la motivation contextuelle et situationnelle sont plus élevés que ceux du groupe contrôle. Sur le plan de la motivation contextuelle, la différence de scores entre les deux groupes à la fin de l'implémentation s'explique principalement par une diminution des scores au niveau de la théorie de l'expectancy-value chez le groupe contrôle, associée à un maintien de la motivation chez le groupe expérimental. Plus précisément, il s'avère que le groupe contrôle a plus fortement diminué au niveau de l'intérêt et de l'utilité perçue pour le cycle d'Acrogym. Dans la théorie de l'expectancy-value, l'intérêt est défini comme l'ensemble des émotions positives ressenties par un individu lorsqu'il réalise une tâche, comme le plaisir ou l'excitation (Fenouillet, 2012). Quant à l'utilité, elle fait référence à l'adéquation d'une activité avec les buts actuels et futurs d'un élève (Eccles \& Wigfield, 2002). Par exemple, un élève peut considérer que la course à pied est utile à partir du moment où il pense que cette activité peut avoir un bénéfice pour son style de vie sain et actif. Nous pouvons ainsi interpréter nos résultats par le fait qu'un cycle d'Acrogym enseigné selon le style de la commande influence négativement l'intérêt des élèves et ne les aide pas à percevoir quelle pourrait être l'utilité de cette activité pour leurs autres activités extrascolaires. Ce résultat n'est pas si surprenant que cela si l'on considère que les élèves n'ont pas eu de choix durant les leçons d'Acrogym et sachant que ce style d'enseignement n'est pas le plus favorable à la motivation intrinsèque des élèves (Papaioannou et al., 2012 ; Pitsi et al., 2015). Enfin plus généralement, le déclin observé chez le groupe contrôle au niveau des espérances de succès et valeurs de la tâche (expectancy-value) est consistant avec les travaux antérieurs. Ces derniers ont montré qu'à l'adolescence, l'auto-évaluation des compétences des élèves est plus juste et réaliste (Wigfield \& Eccles, 2000). Ils sont ainsi plus à même d'évaluer leur succès dans une tâche, ce qui augmente l'importance des espérances de succès dans l'engagement des élèves en EP. De ce fait, si des élèves sont soumis à des styles d'enseignement comme celui de la commande, où il s'agit de répéter le plus fidèlement possible une démonstration opérée par le professeur, alors leurs espérances de succès peuvent diminuer au fil du cycle. Enfin, Yli-Piipari et Kokkonen (2014) avaient également trouvé que l'intérêt, l'importance et les espérances de succès étaient des variables prédictives de l'engagement en EP. Nous pouvons donc en déduire que l'engagement des élèves dans le groupe contrôle a diminué au fur et à mesure du cycle d'Acrogym. 


\section{eJRIEPS Hors Série 3 Décembre 2019}

Au plan de la motivation situationnelle au début du cycle, les élèves du groupe expérimental ont rapporté des scores plus élevés notamment dans trois dimensions de l'IS : la nouveauté, la demande d'attention et le défi (tailles d'effet les plus importantes). Dans la mesure où la majorité des élèves découvrait le style d'enseignement par la découverte guidée et que celui-ci était couplé aux technologies numériques, il est normal que le score pour la nouveauté soit élevé. Nous pouvons également penser que la demande d'attention a été supérieure puisque les élèves ont été soumis à des phases de recherche par l'utilisation des tablettes tactiles et qu'ils devaient se concentrer pour tenter de résoudre le problème posé par le professeur. Enfin, la conjonction d'une demande d'attention supérieure et de la nouveauté a forcément conduit ces élèves à percevoir les leçons d'Acrogym comme plus difficiles. Il s'agit d'un temps d'adaptation à un nouveau style d'enseignement et à l'utilisation des technologies numériques. A la différence du début du cycle, les différences en fin de cycle étaient cette fois significatives pour le plaisir instantané, l'intention d'exploration et la demande d'attention avec des scores plus élevés pour le groupe expérimental. Initialement basées sur la nouveauté, le défi et la demande d'attention au début du cycle, les différences entre les deux groupes sur l'IS se sont donc progressivement dirigées vers le plaisir instantané et l'intention d'exploration (tailles d'effet les plus importantes). Les élèves se sont donc progressivement habitués au style d'enseignement par la découverte guidée et à l'utilisation des technologies numériques. Ils ressentent désormais une immersion dans les leçons d'Acrogym avec le plaisir instantané et l'intention d'exploration. Ces résultats sont congruents avec plusieurs études ayant montré des relations fortes entre le plaisir, l'intérêt et l'exploration de l'information (Ainley \& Ainley, 2011 ; Chen et al., 2001 ; Rotgans \& Schmidt, 2011). Par exemple, Ainley et Ainley (2011) ont démontré des interrelations positives entre le désir d'en apprendre plus à propos d'un sujet en particulier et le sentiment de plaisir en sciences. Dans le même ordre d'idée, Rotgans et Schmidt (2011) ont trouvé que l'IS des élèves était augmenté après la présentation d'un problème à résoudre aux élèves. Nous pouvons donc penser que les activités de résolution de problème auxquelles ont été soumis les élèves du groupe expérimental ont développé leur IS, notamment au niveau de l'intention d'exploration et du plaisir instantané. De plus, l'utilisation des technologies numériques et plus spécifiquement des feedback vidéo ont certainement aidé dans ce processus, puisqu'il a été démontré que ces technologies avaient un impact positif sur la motivation des élèves lorsqu'une pédagogie leur offrait plus d'autonomie et de flexibilité (Fleischer, 2012). Les outils numériques soutenus par le style de la découverte guidée ont donc probablement installé de nouvelles relations pédagogiques entre le 


\section{eJRIEPS Hors Série 3 Décembre 2019}

professeur et ses élèves, et permis aux élèves de développer leur motivation intrinsèque (Engin \& Donanci, 2015 ; Sung, Chang, \& Liu, 2016).

\subsection{Une évolution de la motivation situationnelle au cours du cycle}

Notre deuxième hypothèse est validée dans la mesure où la motivation situationnelle du groupe expérimental a davantage évolué que celle du groupe contrôle. En effet, une évolution mineure a été constatée chez le groupe contrôle avec une baisse du plaisir instantané au cours du cycle, ce qui est cohérent avec la diminution des espérances de succès et valeurs de la tâche constatée post-implémentation. Par conséquent, il apparaît que le style de la commande peine à maintenir la motivation des élèves, tant sur le plan contextuel que situationnel. A l'inverse, une évolution importante a été mesurée chez le groupe expérimental, où les résultats montrent que les élèves ont perçu davantage de plaisir instantané et d'intention d'exploration au fur et à mesure du cycle dans le même temps que la nouveauté et le défi diminuait. En accord avec nos interprétations relatives à la première hypothèse, les scores élevés pour la nouveauté et le défi au début du cycle peuvent s'expliquer par une phase d'adaptation à un nouveau style d'enseignement, d'autant plus que celui-ci était couplé avec des technologies numériques. Il a déjà été démontré dans des études antérieures de fortes corrélations entre la nouveauté et le défi (Roure, LentillonKaestner, Méard \& Pasco, 2019). En effet, les nouveaux contenus d'enseignement sont souvent perçus par les élèves comme étant plus difficiles. Dans la mesure où il semblerait que le niveau de difficulté ait dépassé le sentiment de compétence des élèves au début du cycle, les élèves n'avaient pas encore l'occasion de vraiment s'immerger dans les leçons et ressentir du plaisir instantané et de l'intention d'exploration. Cette interprétation est consistante avec Ding, Sun et Chen (2013) qui ont également trouvé qu'un haut niveau de défi n'était pas forcément positif pour les autres dimensions de l'IS lorsque les élèves reportaient également un haut niveau de nouveauté. En conclusion, cette phase normale d'adaptation s'est fait ressentir au niveau de la nouveauté et du défi, au détriment d'autres dimensions de l'IS. Pour autant la demande d'attention était également bien présente dès le début du cycle. Ceci peut d'expliquer par le fait que les élèves devaient rechercher de l'information, expérimenter différentes solutions et tenter de résoudre un problème lors de leurs leçons d'Acrogym. Ces différentes activités pourraient d'apparenter à un niveau profond de traitement de l'information, celui-ci ayant été régulièrement associé aux tâches de compréhension et d'intégration chez les élèves (Diseth \& Martinsen, 2003 ; Liem, Lau \& 


\section{eJRIEPS Hors Série 3 Décembre 2019}

Nie, 2008). En d'autres termes, les élèves ont perçu une demande d'attention plus élevée de par les activités cognitives qu'ils devaient développer au cours de leur utilisation des technologies numériques au service de la découverte guidée. L'évolution en fin de cycle caractérisée par la baisse du défi témoigne de l'adaptation réussie des élèves avec le style d'enseignement par la découverte guidée. Cette adaptation s'est probablement réalisée en partie grâce au rôle actif joué par l'enseignante, dans la mesure où cette dernière servait de guide aux élèves pour les amener à trouver la solution. Les élèves se sont progressivement habitués à cette nouvelle relation avec l'enseignante pour enfin percevoir les activités comme moins difficiles qu'au début du cycle. De plus, l'utilisation des technologies numériques a pu aussi contribuer à faire en sorte que les tâches proposées soient de difficulté optimale, grâce à l'emploi des feedback vidéo et des commentaires des camarades et du professeur. En effet, ces différents commentaires ont certainement réduit la charge cognitive des élèves associée à l'exploitation des vidéos produites lorsque les élèves essayaient d'analyser les pyramides construites (Spanjers, Van gog \& Van Merriënboer, 2010). Enfin, la fin du cycle a permis aux élèves de pleinement s'immerger dans les leçons d'Acrogym et ressentir du plaisir instantané et de l'intention d'exploration. Ce résultat obtenu en fin de cycle pour la motivation situationnelle est très intéressant et permet de renforcer des résultats obtenus dans de précédentes études. Par exemple Chen, Darst et Pangrazi (2001) avaient eux aussi mis en évidence que plutôt que de proposer des situations nouvelles et différentes aux élèves, il était plus intéressant de construire des situations suscitant l'intention d'exploration et une demande cognitive élevée si les enseignants souhaitaient augmenter le plaisir instantané des élèves et leur IS. Plusieurs recherches ont d'ailleurs démontré que les processus cognitifs de haut niveau demandant une exploration active de l'environnement, associés au plaisir, représentaient des facteurs d'engagement des élèves dans les leçons d'EP (Jaakkola, Wang, Soini \& Liukkonen, 2015 ; Roure \& Pasco, 2018b).

\section{Conclusion, limites et perspectives}

En conclusion, l'utilisation des technologies numériques couplée au style d'enseignement par la découverte guidée semble être une option intéressante pour développer l'IS des élèves, et plus largement leur engagement en EP. Toutefois, les résultats de cette étude doivent être interprétés avec précaution dus à deux limites principales. Premièrement, le groupe contrôle utilisé lors de cette recherche a suivi un enseignement selon le style de la 


\section{eJRIEPS Hors Série 3 Décembre 2019}

commande sans outils numériques. Les différences constatées avec le groupe expérimental sont donc à la fois dues au style d'enseignement par la découverte guidée et à l'utilisation des technologies numériques. Nous avions fait ce choix car la littérature indiquait que les bénéfices des technologies numériques devaient être étudiés à partir d'une approche pédagogique (Casey, Goodyear \& Armour, 2017). Toutefois, de futures études pourraient utiliser des conditions de contrôle alternatives, comme un groupe avec les technologies numériques et le style de la commande, ou un groupe avec le style de la découverte guidée mais sans les technologies numériques. Cependant, il est utile de rappeler que les recherches conduites dans un contexte écologique de classe doivent prendre en compte les contraintes de l'enseignement de l'EP dans les classes, et qu'il est souvent difficile de se rapprocher de conditions idéales (comme en laboratoire). La deuxième limite provient de l'échantillon exclusivement féminin, induit par les classes dont disposait l'enseignante participante. II serait intéressant de développer des recherches similaires dans des classes d'EP mixtes afin de prendre comme variable d'étude supplémentaire le sexe des élèves.

\section{Bibliographie}

Ainley, M., \& Ainley, J. (2011). Student engagement with science in early adolescence: The contribution of enjoyment to students' continuing interest in learning about science. Contemporary Educational Psychology, 36(1), 4-12. doi: 10.1016/j.cedpsych.2010.08.001.

Banville, D., \& Polifko, M.F. (2009). Using digital video recorders in physical education. Journal of Physical Education, Recreation \& Dance, 80(1), 17-21.

Casey, A., \& Jones, B. (2011). Using digital technology to enhance student engagement in physical education. Asia-Pacific Journal of Health, Sport and Physical Education, 2(2), 51-66.

Casey, A., Goodyear, V.A., \& Armour, K.M. (2017). Digital technologies and learning in physical education. New York: Routledge.

Chatzipanteli, A., Digelidis, N., \& Papaioannou, A. (2015). Self-regulation, motivation and teaching styles in physical education classes: An intervention study. Journal of Teaching Physical Education, 34, 333-344.

Chen, A., Darst, P.W., \& Pangrazi, R. (2001). An examination of situational interest and its sources. British Journal of Educational Psychology, 71, 385-400. 


\section{eJRIEPS Hors Série 3 Décembre 2019}

Dania, A., Hatziharistos, D., Koutsouba, M., \& Tyrovola, V. (2011). The use of technology in movement and dance education: recent practices and future perspectives. Procedia Social and Behavioral Sciences, 15, 3355-3361.

Ding, H., Sun, H., \& Chen, A. (2013). Impact of expectancy-value and situational interest motivation specificity on physical education outcomes. Journal of Teaching in Physical Education, 32, 253-269.

Diseth, A., \& Martinsen, O. (2003). Approaches to learning, cognitive style, and motives as predictors of academic achievement. Educational Psychology, 23(2), 195-207.

Eccles, J.S., \& Wigfield, A. (2002). Motivational beliefs, values, and goals. Annual Review of Psychology, 53, 109-132. doi:10.1146/annurev.psych.53.100901.135153

Elliot, A.J., \& McGregor, H.A. (2001). A 2 X 2 Achievement goal framework. Journal of Personality and Social Psychology, 80, 501-519.

Engin, M., \& Donanci, S. (2015). Dialogic teaching and iPads in the EAP classroom. Computers \& Education, 88, 268-279.

Fédération Wallonie-Bruxelles (2015). Indice socio-économique des écoles en Fédération Wallonie Bruxelles. Retrieved from https://fusiontables.googleusercontent.com/emb edviz?q=select+col7+from+1 cupk7E14ePvCcTyf8bdE6rqtW35MtfA5O2_VAbeO\&vi $z=M A P \& h=$ false $\& l a t=50.337291139121405 \& \operatorname{lng}=4.481085245556642 \& t=1 \& z=9 \& l=$ col7\&y=2\&tmplt=2\&hml=ONE_COL_LAT_LNG

Fenouillet, F. (2012). Les théories de la motivation. Paris : Dunod.

Fleischer, H. (2012). What is our current understanding of one-to-one computer projects: A systematic narrative research review. Educational Research Review, 7, 107-122.

Fullan, M. (2013). Stratosphere: Integrating Technology, Pedagogy, and Change Knowledge. Toronto: Pearson.

Godeau E., Navarro F., \& Arnaud C. (2012). La santé des collégiens en France / 2010. Données françaises de l'enquête internationale Health Behaviour in School-aged Children (HBSC). Saint-Denis : Inpes, coll. Études santé.

Goodyear, V.-A, Casey, A., \& Kirk, D. (2014). Hiding behind the camera: Social learning within the cooperative learning model to engage girls in physical education. Sport, Education and Society, 19(6), 712-734.

Harris, F. (2009). Visual technology in physical education using Dartfish video analysis to enhance learning: An overview of the Dartfish project in New Brunswick. Physical \& Health Education Journal, 74(4), 24-25. 


\section{eJRIEPS Hors Série 3 Décembre 2019}

Harvey, S., \& Gittins, C. (2014). Effects of integrating video-based feedback into a teaching games for understanding soccer unit. Agora for Physical Education and Sport, 16(3), 271-290.

Huff, M., \& Schwan, S. (2012). The verbal facilitation effect in learning to tie nautical knots. Learning and Instruction, 22(5), 376-385.

Jaakkola, T., Wang, J., Soini, M., \& Liukkonen, J. (2015). Students' perceptions of motivational climate and enjoyment in Finnish physical education: A latent profile analysis. Journal of Sports Science and Medicine, 14, 477-483.

Kirby, S., Byra, M., Readdy, T., \& Wallhead, T. (2015). Effects of spectrum teaching styles on college students' psychological needs satisfaction and self-determined motivation. European Physical Education Review, 21(4), 521-540.

Korn, R.M., \& Elliot, A.J. (2016). The 2× 2 Standpoints Model of Achievement Goals. Frontiers in Psychology, 7: 742. doi: 10.3389/fpsyg.2016.00742

Kretschmann, R. (2015). Effect of physical education teachers' computer literacy on technology use in physical education. Physical Educator, 72(5), 261-277.

Legrain, P., Gillet, N., Gernigon, C., \& Lafreniere, M.-A (2015). Integration of information and communication technology and pupils' motivation in a physical education setting. Journal of Teaching in Physical Education, 34(3), 384-401.

Liem, A.D., Lau, S., \& Nie, Y. (2008). The role of self-efficacy, task value, and achievement goals in predicting learning strategies, task disengagement, peer relationship, and achievement outcome. Contemporary Educational Psychology, 33(4), 486-512.

Lu-Ho, H., Iwen, H., \& Gwo-Jen, H. (2016). Effects of different online peer-feedback approaches on students' performance skills, motivation and self-efficacy in a dance course. Computers \& Education, 96, 55-71.

Morgan, K., Kingston, K., \& Sproule, J. (2005). Effects of different teaching styles on the teacher behaviours that influence motivational climate and pupils' motivation in physical education. European Physical Education Review, 11(3), 257-285.

Mosston, M., \& Ashworth, S. (2002). Teaching physical education (5 $5^{\text {th }}$ ed.). San Francisco, CA: Benjamin Cummings.

O'Loughlin, J., Chroinin, D.-N., \& O'Grady, D. (2013). Digital video: The impact on children's learning experiences in primary physical education. European Physical Education Review, 19(2), 165-182. 


\section{eJRIEPS Hors Série 3 Décembre 2019}

Palao, J.M., Hastie, P.A., Cruz, P.G., \& Ortega, E. (2015). The impact of video technology on student performance in physical education. Technology, Pedagogy and Education, 24(1), 51-63.

Papaioannou, A, Theodosiou, A., Pashali, M., \& Digelidis, N., \&. (2012). Advancing task involvement, intrinsic motivation and metacognitive regulation in physical education classes: The self-check style of teaching makes a difference. Advance in Physical Education, 2(3), 110-118.

Pitsi, A., Digelidis, N., \& Papaioannou, A. (2015). The effects of reciprocal and self-check teaching styles in students' intrinsic-extrinsic motivation, enjoyment and autonomy in teaching traditional Greek dances. Journal of Physical Education and Sport, 15(2), 352-361.

Preacher, K.J., Zhang, Z., \& Zyphur, M.J. (2011). Alternative methods for assessing mediation in multilevel data: the advantages of multilevel SEM. Structural Equation Modeling: A Multidisciplinary Journal, 18(2), 161-182.

Raiola, G., Parisi, F., \& Napolitano, S. (2014). Sports skills in youth volleyball video analysis teaching method. Procedia - Social and Behavioral Sciences, 117, 436-441.

Ream, G.L., Elliott, L.C., \& Dunlap, E. (2013). Trends in video game play through childhood, adolescence, and emerging adulthood. Psychiatry journal, 2013(1), 7-28.

Riou, F., Boiché, J., Doron, J., Romain, A.J., Corrion, K., Ninot, G., et al. (2012). Development and validation of the French achievement goals questionnaire for sport and exercise (FAGQSE). European Journal of Psychological Assessment, 28(4), 313-320.

Rotgans, J.L., \& Schmidt, H.G. (2011). Situational interest and academic achievement in the active-learning classroom. Learning and Instruction, 21(1), 58-67. doi: 10.1016/j.learninstruc.2009.11.001.

Roure, C., \& Lentillon-Kaestner, V. (2018). Development, validity and reliability of a French expectancy-value questionnaire in physical education. Canadian Journal of Behavioural Science, 50 (3), 127-135. doi: 10.1037/cbs0000099.

Roure, C., Lentillon-Kaestner, V., Méard, J., \& Pasco, D. (2019). Universality and uniqueness of students' situational interest in physical education: A comparative study. Psychologica Belgica, 59(1), 1-15.

Roure, C., \& Pasco, D. (2018a). Exploring situational interest sources in the French physical education context. European Physical Education Review, 24(1), 3-20. doi: $10.1177 / 1356336 \times 16662289$ 


\section{eJRIEPS Hors Série 3 Décembre 2019}

Roure, C., \& Pasco, D. (2018b). The impact of learning task design on students' situational interest in physical education. Journal of Teaching in Physical Education, 37, 24-34. doi: 10.1123/jtpe.2017-0046

Roure, C., Pasco, D., \& Kermarrec, G. (2016). Validation de l'échelle française mesurant l'intérêt en situation, en éducation physique. Revue Canadienne des sciences du comportement, 48(2), 112-120.

Salvara, M.I., Jess, M., Abbott, A., \& Bognar, J. (2006). A preliminary study to investigate the influence of different teaching styles on pupils' goals orientations in physical education. European Physical Education Review, 12(1), 51-74.

Selwyn, N. (2014). Distrusting educational technology: Critical questions for changing times. London: Routledge.

Spanjers, I., van Gog, T., \& van Merriënboer, J. (2010). A theoretical analysis of how segmentation of dynamic visualizations optimizes students' learning. Educational Psychology Review, 22, 411-423.

Stillar, B. (2012). Twenty first century learning: how college classroom interaction will change in the decades ahead. The International Journal of Technology, Knowledge, and Society, 8(1), 143-151.

Sung, Y.T., Chang, K.E., \& Liu, T.C. (2016). The effects of integrating mobile devices with teaching and learning on students' learning performance: A meta-analysis and research synthesis, Computers \& Education, 94, 252-275.

Weir, T., \& O'Connor, S. (2009). The use of digital video in physical education. Technology, Pedagogy and Education, 18(2), 155-171.

Wigfield, A., \& Eccles, J.S. (2000). Expectancy-value theory of achievement motivation. Contemporary Educational Psychology, 25(1), 68-81.

Xiang, P., Liu, Y., McBride, R.E., \& Bruene, A. (2011). Longitudinal goal patterns and their effects on students' motivation in running programs. The Journal of Experimental Education, 79(3), 295-317.

Yli-Piipari, S., \& Kokkonen, J. (2014). An application of the expectancy-value model to understand adolescents' performance and engagement in physical education. Journal of Teaching in Physical Education, 33(2), 250-268. 